\title{
Ecological niche modeling of some Costa Rican myxomycetes
}

\author{
Rojas C ${ }^{1,2 *}$, Zúñiga JM', Stephenson $\mathrm{SL}^{3}$
}

\author{
${ }^{1}$ Forest Resources Unit, Engineering Research Institute, University of Costa Rica, San Pedro de Montes de Oca, \\ 11501 Costa Rica \\ ${ }^{2}$ Department of Agricultural Engineering, University of Costa Rica, San Pedro de Montes de Oca, 11501-Costa Rica \\ ${ }^{3}$ Department of Biological Sciences, University of Arkansas, Fayetteville AR 72701, United States of America
}

Rojas C, Zúñiga JM, Stephenson SL 2015 - Ecological niche modeling of some Costa Rican myxomycetes. Current Research in Environmental \& Applied Mycology 5(2), 153-159, Doi $10.5943 /$ cream/5/2/10

\begin{abstract}
Ecological niche modeling has not been used extensively in studies of myxomycetes but can provide important information for research efforts to develop a better understanding of the ecology of these organisms. With a dataset of myxomycete records for the five most commonly recorded species in Costa Rica, a series of probability distribution models was generated using the maximum entropy technique. The latter was carried out with the objective of evaluating the usefulness of such protocols to provide feedback for future research. Models showed Arcyria cinerea and Didymium iridis to have wider but more moderate probability distributions in comparison with Physarum compressum, Didymium squamulosum and Hemitrichia calyculata. Elevation was the variable that contributed the most to all ecological niche models, but a greater number of studies have been carried out in Costa Rica at high elevations. As such, it seems that the models showed the need for low elevation systematic surveys more realistically than providing a representation of the niche and distribution for the species being considered. However, this study does serves as baseline for future assessments of myxomycete distribution patterns in the framework of climate change.
\end{abstract}

Key words - biodiversity informatics - biogeography - Central America - distribution myxogastrids

\section{Introduction}

In ecological theory, the conceptual difference between the fundamental and the realized niche is related to the level of resource utilization after biological interactions and dispersal barriers are considered (see Hutchinson 1957, Tingley et al. 2014). In the first instance, species display their full potential, whereas in the second instance species are limited in their ability to use resources depending on the strength and direction of external factors. Under the global conditions of the XXI century, some theorists also have proposed that in addition to the two concepts mentioned above, a more applied set of categories, including risk-related niches, can be defined as well (e.g. Sax et al. 2013). Such a modern approach, which has been basically proposed for monitoring species-systems interactions, requires both the rapid generation of data and the use of rapid assessment projects to generate information about understudied groups.

It is under the framework of the latter concept that the use of ecological niche modeling, an application of bioinformatics, may help generate much needed information about the distribution of 
the majority of species on the planet. Considering that species distribution information already provides data relating to the interaction of species and the environment, such information is far from trivial. For this reason, niche modeling techniques also have been used to assess niche stability (Stigall 2012), to monitor species over time (Broennimann et al. 2012), to assess species ecological potential (Tingley et al. 2014) and to make management decisions in the context of future climate change scenarios (see Gelviz-Gelvez et al. 2015).

Focusing on the myxomycetes, a well-recognized group of amoeboid protists known to inhabit the entire planet (Stephenson 2003), niche modeling has not been widely used in literature. One recent exception is the work of Aguilar et al (2014). Even though there are several reasons why the latter has not taken place, perhaps the most important one is the limited amount of quality information for the majority of geographical areas in the world. Some temperate localities may have data that can be incorporated into a niche model, but most tropical examples still require stronger in situ distributional sets. In the Neotropics, for instance, there are few areas where niche modeling can be carried out, and Costa Rica seems to be one of those. For this country, there are records of myxomycetes from the early twentieth century (see Rojas \& Doss 2013), efforts to develop consolidated lists of species and records that began in the 1970's (Alexopoulos \& Sáenz 1975), studies on species distribution within forest types (see Schnittler \& Stephenson 2000) and distributional information about myxomycetes in poorly studied tropical ecosystems such as the paramo and the dry forest (see Rojas et al. 2010a, 2010b). In this way, Costa Rica provides a dataset that is geographically extensive enough and temporally strong enough for niche modeling examination.

It is for the latter reason that the present rapid study was designed. The objective of this project was to evaluate, using a niche modeling technique, the probability distribution of the five most commonly recorded species of myxomycetes in Costa Rica in order to analyze the capacity of such technique to offer quality information about the distribution of members of this group of organisms within this country. The fundamental idea behind this project is that niche modeling may provide input about the status of myxomycete studies in a particular geographical area and may be used for species monitoring and to develop more refined ecological hypotheses about the group.

\section{Materials \& Methods}

The present study used the distributional information of approximately 2000 records from 101 localities corresponding to the five most commonly recorded species of Costa Rican myxomycetes obtained during the period between 1905 and 2014 (see Fig. 1). The source of the records prior to 2009 is summarized in Rojas et al. (2010a), and information from the last five years of the studied period was obtained directly by all three authors using both field and laboratory techniques described by Stephenson \& Stempen (1994). The reason for selecting only commonly recorded species is explained by Phillips et al. (2006) and is related to the learning capability of modeling software, which decreases error when a stronger distributional input dataset is used.

For older records with no geographical information recorded originally, a calculation of the geographic centroid was performed by examining the closest known locality and the original survey routes using the original field information from herbaria where vouchers were deposited. Information for records after 1990 was obtained directly from collectors. Once the database was compiled and purged for errors, all geographical coordinates were checked to correspond to the CR05 official datum for Costa Rica. For the present study, the taxonomic treatment followed is that of Lado (2005-2015), and the morphological concept of species was used.

To obtain model variables for biophysical analysis, a series of bioclimatic and elevational datasets was obtained from WorldClim (www.worldclim.org). This information was used to create 30 second resolution raster datasets that were geographically restricted using vectorial information from the 2014 Digital Atlas of Costa Rica (www.tec.ac.cr). The latter protocol generated country wide biophysical datasets with a cell resolution of about $1 \mathrm{~km}^{2}$. 
The ecological niche models were performed using the maximum entropy technique in MaxEnt, version 3.3.3 (see Phillips et al. 2006 and Elith et al. 2011 for theoretical considerations) using the datasets for elevation, isothermality, annual temperature range, annual precipitation and the coefficient of precipitation variation as biophysical variables. The respective known geographical distribution for each of the five selected species was used as the input set for the model. A jackknife test and logistic output were also used in order to evaluate the importance of each biophysical variable to model robustness after 20 replicated model runs.

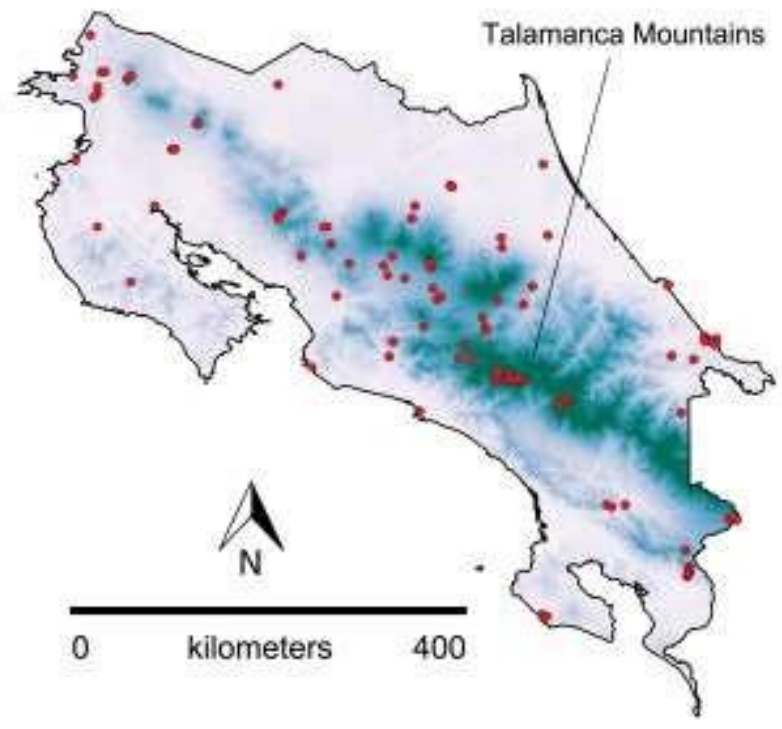

Fig. 1 - Map of the continental section of Costa Rica showing localities (as red dots) where records of the five most commonly recorded myxomycetes have been obtained during the period 19052014. The darker areas correspond to higher elevations such as the Talamanca Mountain range.

The output datasets of average probability distribution were imported into ArcMap 10.1, where a modified raster using the delimitation of the Holdridge Life Zones for Costa Rica was created for clearer visualization of results. For the latter, a simplified probability distribution display option was selected by establishing four categories defined by quartile-based cutoff values. In this way, very low probability (VLP) was defined for values between 0.00 and 0.24 , low probability (LP) for values between 0.25 and 0.49 , medium probability (MP) for values between 0.50 and 0.74 and high probability (HP) for values between 0.75 and 1.00 .

For the obtained niche models, the receiver operating characteristic (ROC) curves were analyzed in terms of the area under the curve (AUC) as suggested by Phillips et al. (2006). The ROC curves provide a characterization of the performance of the models in all possible conditions of raster cell determination and the AUC permits a rapid across-model evaluation of such performance. The latter value oscillates between 0.5 and 1.0, with the lower value being assigned to chance and the higher one to an accurate discrimination of category options, which in the case of an environmental niche model corresponds to presence or absence of the species in a particular raster cell.

\section{Results}

The five most commonly recorded species of myxomycetes in Costa Rica were Arcyria cinerea (Bull.) Pers. with 747 records, Physarum compressum (Alb. \& Schwein.) Kuntze with 310 records, Didymium squamulosum (Alb. \& Schwein) Fr. \& Palmquist with 303 records, Didymium iridis (Ditmar) Fr. with 277 records and Hemitrichia calyculata (Speg.) M. L. Farr with 228 records. Most of the records for all of these species were obtained in the last 25 years and were 
collected primarily from lignicolous substrates on the forest floor or with the use of moist chamber cultures under laboratory conditions.

All niche models were moderately robust in terms of their ROC curves, with AUC values between 0.796 and 0.841 . In Table 1 it can be noted that for all species, the biophysical variable with the largest contribution to the respective niche model was elevation. The second most important variables were isothermality and annual temperature range. The least important variable in all cases was annual precipitation. Results from the jackknife evaluation showed that elevation was the variable that contained the most information for the construction of the models.

Table 1 Average contribution (\%) values for all biophysical variables used in the niche models for the five analyzed myxomycete species, based on logistic response curves.

\begin{tabular}{lccccc}
\hline Species & \multicolumn{3}{c}{ Biophysical variable } \\
& Elevation & Isothermality & $\begin{array}{c}\text { Temperature } \\
\text { Range }\end{array}$ & Precipitation & $\begin{array}{c}\text { Coefficient of } \\
\text { Precipitation }\end{array}$ \\
\hline Arcyria cinerea & 60.5 & 11.0 & 12.4 & 4.8 & 11.3 \\
Physarum compressum & 63.2 & 15.8 & 5.2 & 7.7 & 8.1 \\
Didymium squamulosum & 72.6 & 9.4 & 7.3 & 2.1 & 8.7 \\
Didymium iridis & 48.9 & 20.4 & 6.6 & 4.9 & 19.2 \\
Hemitrichia calyculata & 62.9 & 5.8 & 5.4 & 7.5 & 18.4 \\
\hline
\end{tabular}

The environmental niche models for the five selected species (Fig. 2) showed different results each example being considered. Both $A$. cinerea and $D$. iridis were predicted to have a broader geographical distribution associated with moderate probability values, whereas $P$. compressum, $D$. squamulosum and $H$. calyculata showed the largest areas, with very low probabilities. In the case of the last species, the model showed the largest area with a high probability of occurrence.

The areas in the country with the lowest probability of occurrence for all five species combined were the northeastern and eastern Caribbean and the south Pacific. Interestingly, different sections of the Talamanca Range in the central-southern region of Costa Rica showed the highest probability of occurrence in every instance.

\section{Discussion}

The results from the ecological niche models for the five most frequently recorded species of myxomycetes in Costa Rica should be interpreted with caution. As is the case with any type of application of bioinformatics, direct results alone may not provide a strong enough framework for the development of conclusions. In this manner, the level of correspondence of obtained results with other data generated using different techniques should always be practiced. Moreover, as seen in Aguilar et al. (2014) a different interpretation of the species concept may change dramatically the potential distribution generated using the environmental niche modeling techniques.

In this context, it is should be mentioned that Rojas et al. (2010b) had already reported that elevation seemed to be the most important variable explaining the distribution of myxomycetes in Costa Rica. These authors also noted that $H$. calyculata and $D$. squamulosum had been preferentially recorded in premontane forests as supposed to lowland forests, where the other three species considered herein have been more commonly encountered. Whether or not those observations actually correspond with a real preference for forest types and elevations is outside the scope of the present study but it is certainly represents a topic that can be more carefully investigated with a systematic survey across a defined geographical area and re-evaluation using computational models such as the ones used in this study.

Similarly, the issue of elevation being such an important variable explaining the distribution of myxomycetes in Costa Rica probably relates in part to the intensive collecting effort carried out 
in the last 40 years (e.g., Alexopoulos and Sáenz 1975, Schnittler \& Stephenson 2000, Rojas \& Stephenson 2007, Rojas et al. 2011) that has targeted high-elevation areas in the country. Since most of the myxomycete records for Costa Rica were obtained during that period of time (see Rojas et al. 2010a), it seems intuitive to think that a computational model would show species to be associated with high elevations as a product of effort and survey location and not necessarily be due to species preference. Interestingly, the exclusion of higher elevation subdatasets during analysis also showed a noticeable difference on the probability associated with non-mountainous areas (not shown) and thus indicated that the other biophysical variables may have been playing an equivalent role in shaping distributions. In the results obtained in the present study, the latter situation could have been the case for $D$. iridis, based on the values presented in Table 1 .

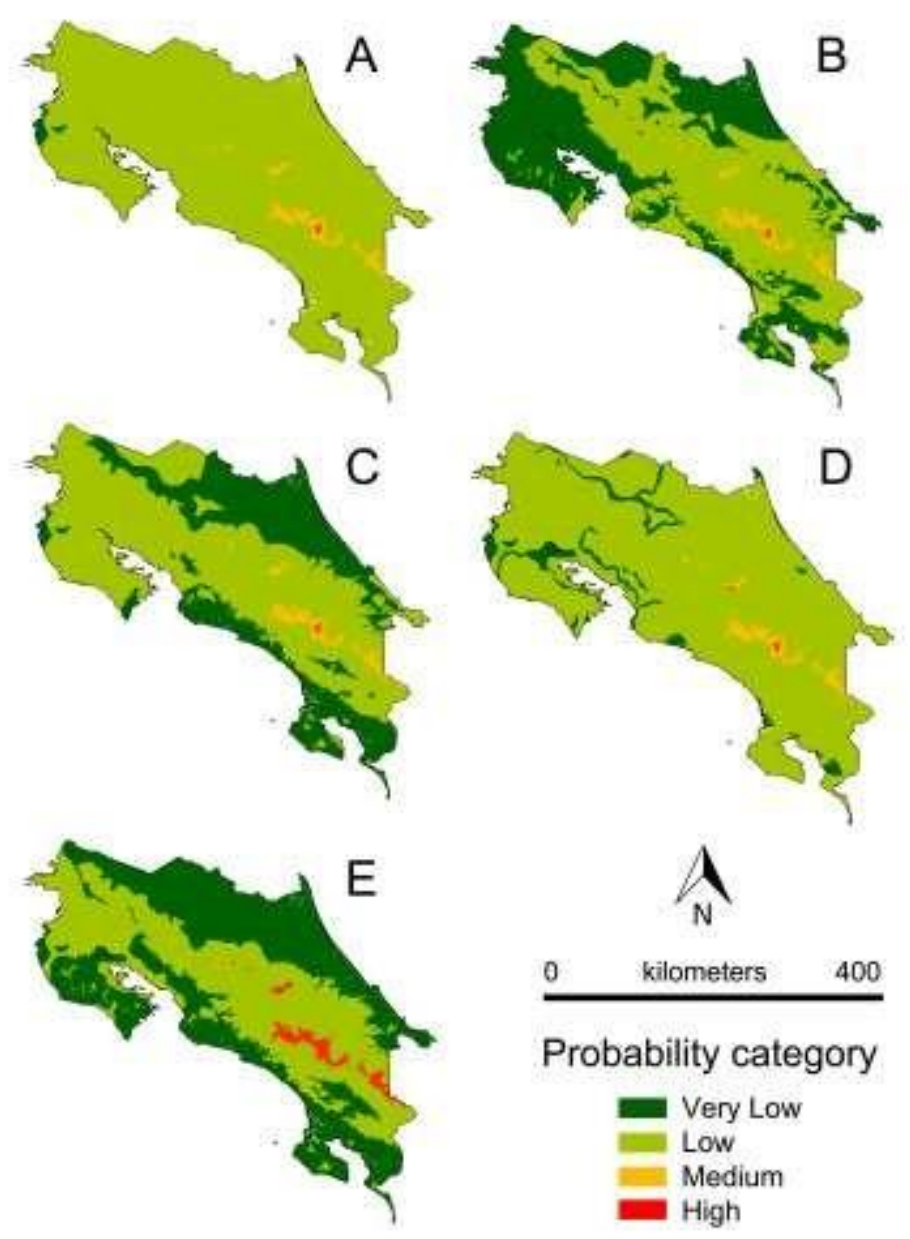

Fig. 1 - Maximum entropy-based environmental niche models for the most commonly recorded species of myxomycetes in Costa Rica between 1905 and 2014. (A) Arcyria cinerea, (B) Physarum compressum, (C) Didymium squamulosum, (D) Didymium iridis and (E) Hemitrichia calyculata. For an explanation of the probability categories see Materials \& Methods.

Although both isothermality and the annual temperature range may have a direct impact on myxomycete life cycles, very few empirical studies using tropical material have been carried out under realistic conditions. Most of the classical laboratory studies were carried out at least 50 years ago in situations associated with temperate regions (see Gray \& Alexopoulos 1968) and may not reflect the possibly quite different dynamics associated with tropical areas of the world. Fortunately, the topic is capturing the attention of some researchers again, and it is likely that in the near future some published across-regions data can be used to reinterpret basic natural history observations for myxomycetes. In any case, such information, potentially captured by the niche models presented 
herein is important to evaluate ecological concepts and their relevance to myxomycete analysis (i.e., what geographical scales are useful for addressing which ecological questions?).

Independently to the last observation, the power of the maximum entropy approach to calculate potential distribution based on historical data is remarkable. However, as it has been stated by a number of authors (e.g., Broennimann et al. 2012), its full potential may be observed only when a systematic study of a particular taxonomic group is carried out over both a geographical and a temporal range. For myxomycete research, this technique may represent an important tool for analysis of information in relation to a number of ecological issues affecting the occurrence and potential distribution of species. Once again, this information may help develop more robust and evidence-based applied ecological hypotheses, such as the delimitation and use of niche concepts for myxomycetes.

In spite of the latter, the ecological niche modeling carried out in the present study has shown the need to obtain more information from some regions in Costa Rica. In particular, the Caribbean and the south Pacific areas seem to require more study. Even though for the latter, recent efforts have been carried out and a number of interesting observations have been recorded (but not yet published), additional surveys should be carried out in both currently understudied areas. Such a strategy would first allow an accumulation of localized geographical information on poorly studied assemblages of myxomycetes, and also would be useful for testing the response of environmental niche models to the inclusion of new subdatasets (i.e. molecular-based, see Aguilar et al. 2014). A potential convergence towards a common geographically displayed model, which is the main goal of niche modeling, would permit researchers to allocate resources towards relevant research questions in future studies.

\section{Acknowledgements}

This study was carried out in the context of projects 731-B4-072 and 731-B5-062, both funded by the Vicerrectoría de Investigación of the University of Costa Rica. A number of records of myxomycetes from Costa Rica were obtained with funding provided by the National Science Foundation through grant DEB-0316284 (to SLS).

\section{References}

Aguilar M, Fiore-Donno AM, Lado C, Cavalier-Smith T - Using environmental niche models to test the 'everything is everywhere' hypothesis for Badhamia. The ISME Journal 8, 737745.

Alexopoulos CJ, Sáenz JA. 1975 - The myxomycetes of Costa Rica. Mycotaxon 2, 223-271.

Broennimann O, Fitzpatrick MC, Pearman PB, Petitpierre B, Pellissier L, Yoccoz NG, Thuiller W, Fortin MJ, Randin C, Zimmermann NE, Graham CH, Guisan A. 2012 - Measuring ecological niche overlap from occurrence and spatial environmental data. Global Ecology and Biogeography 21, 481-497.

Elith J, Phillips SJ, Hastie T, Dudík M, Chee YE, Yates CJ. 2011 - A statistical explanation of MaxEnt for ecologists. Diversity and Distributions 17, 43-57.

Hutchinson GE 1957 - Concluding Remarks. Cold Spring Harbor Symposia on Quantitative Biology 22, 415-427.

Gelviz-Gelvez SM, Pavón NP, Illoldi-Rangel P, Ballesteros-Barrera C. 2015 - Ecological niche modeling under climate change to select shrubs for ecological restoration in Central Mexico.Ecological Engineering 74, 302-309.

Gray WD, Alexopoulos CJ. 1968 - Biology of the Myxomycetes. The Ronald Press Company, New York.

Lado C 2005-2015 An on line nomenclatural information system of Eumycetozoa. Real Jardín Botánico, Spain. http://eumycetozoa.com (accessed 15 Feb 2015). 
Phillips SJ, Anderson RP, Schapire RE. 2006 - Maximum entropy modeling of species geographic distributions. Ecological Modelling 190, 231-259.

Rojas C, Stephenson SL. 2007 - Distribution and ecology of myxomycetes in the high-elevation oak forests of Cerro Bellavista, Costa Rica. Mycologia 99, 534-543.

Rojas C, Schnittler M, Stephenson SL. 2010a - A review of the Costa Rican myxomycetes. Brenesia 73-74, 39-57.

Rojas C, Valverde R, Stephenson SL, Vargas MJ. 2010b - Ecological patterns of Costa Rican myxomycetes. Fungal Ecology 3, 139-147.

Rojas C, Stephenson SL, Huxel GR. 2011 - Macroecology of high-elevation myxomycete assemblages in the northern Neotropics. Mycological Progress 10, 423-437.

Rojas C, Doss RG. 2013 - Brief research history and status of myxomycete conservation in the Neotropics. Brenesia 79, 37-43.

Sax DF, Early R, Bellemare J. 2013 - Niche syndromes, species extinction risks, and management under climate change. Trends in Ecology and Evolution 28, 157-523.

Schnittler M, Stephenson SL. 2000 - Myxomycete biodiversity in four different forest types in Costa Rica. Mycologia 92, 626-637.

Stephenson SL, Stempen H. 1994 - Myxomycetes: a handbook of slime molds. Timber Press, Oregon.

Stephenson SL 2003 - The Fungi of New Zealand Volume 3: Myxomycetes of New Zealand. Fungal Diversity Press, Thailand.

Stigall AL 2012 - Using ecological niche modelling to evaluate niche stability in deep time. Journal of Biogeography 39, 772-781.

Tingley R, Vallinoto M, Sequeira F, Kearney MR. 2014 - Realized niche shift during a global biological invasion. PNAS 111, 10233-10238. 